\title{
U17 ve U19 Erkek Milli Badmintoncuların Müsabaka Ortamında Yaptıkları Basit Hataların Karşılaştırılması
}

\author{
Beyhan ÖZGÜR ${ }^{*}$ \\ ${ }^{1}$ Mardin Artuklu Üniversitesi, BESYO, https://orcid.org/0000-0002-7813-4119
}

Öz

Bu çalışmada 17 ve 19 yaş grubu erkek milli badmintoncuların maç ortamında yaptıkları basit hataların karşılaştırılması amaçlanmıştır. Araştırmaya, U19 kategorisinden yaş ortalamas1 $17,39 \pm 0,50$ y1l olan 18 ve U17 kategorisinden yaş ortalaması $15,52 \pm 0,51$ yıl olan 21 erkek milli Badminton oyuncusu gönüllü olarak katılmıştır. Basit hata analizi için U19 kategorisinde 35 tek erkek maçı ve U17 kategorisinde 31 tek erkek maçı değerlendirilmiştir. Basit hatalar, TH (toplamda yapılan basit hata), KTS (kaybedilen toplam sayı), BKTSY (basit hataların kaybedilen toplam sayıdaki yüzdesi), HÖK (hata ön kort), HOK (hata orta kort), HAK (hata arka kort), HAÖK (hata aut ön kort), HAOK (hata aut orta kort), HAAK (hata aut arka kort), TAH (toplamda aut hatas1), HFÖK (hata file ön kort), HFOK (hata file orta kort), HFAK (hata file arka kort), TFH (Toplamda File Hatası) olarak basit hata analiz tablosuna kaydedilmiştir. Elde edilen veriler SPSS 22 paket programına aktarılmış ve tanımlayıcı istatistikleri için minimum, maksimum, ortalama ve standart sapma değerleri hesaplanmıştır. Kategoriler arası ve kazanan ve kazanamayan sporcular arası hatalara ait farkın analizi için Mann Withney U testi kullanılmıștır. U17 ve U19 yaş gruplarının yaptıkları tüm basit hata değerleri benzer bulunmuştur $(\mathrm{p}>0.05)$. U17 kategorisinde kazanan ve kazanamanyan sporcuların KTS değerleri arasında anlamlı fark bulunmuştur $(\mathrm{p}<0.01)$. U19 kategorisinde kazanan ve kazanamanyan sporcuların KTS $(\mathrm{p}<0.01)$, TH, TAH ve HAOK değerleri arasında anlamlı fark bulunmuştur $(\mathrm{p}<0.05)$. Çalışma sonucunda, U19 grubunda, orta kort bölgesinde aut hatası (HAOK), toplamda aut hatası (TAH) ve toplam hata $(\mathrm{TH})$ değerleri daha az olan sporcuların müsabakaları galip olarak bitirdikleri bulunmuştur.

\author{
Orijinal Makale \\ Yayın Bilgisi \\ Gönderi Tarihi: 20.10 .2018 \\ Kabul Tarihi: 21.12.2018 \\ Online Yayın Tarihi: 31.12 .2018
}

DOI: $10.30769 /$ usbd.472815

\section{Comparison of Unforced Errors during Competition of U17 and U19 Male National Badminton Players'}

\begin{abstract}
In this study, it is aimed to compare the unforced errors of the U17 and U19 male national badminton players during the competition. 18 male badminton players from the U19 category (age average 17,39 $\pm 0,50$ ) and 20 male badminton players from the U17 category (age average $15,52 \pm 0,51$ ) participated in the study voluntarily. For simple error analysis, 35 men single matches in the U19 category and 31 men single matches in the U17 category were evaluated. Unforced errors were recorder in the unforced error table as TE ( error in total), TPL (total point lost), ETLPP (unforced error percentage of the total number lost ), EFC (error in front court), EMC (error in mid court), EBC (error in back court), AEFC ( aut error in front court), AEMC (aut error in mid court), AEBC (aut error in back court), TAE ( aut error in total), NEFC ( net error in front court), NEMC (net error in mid court), NEBC (net error in back court), TNE ( net error in total). During the competition, the matches of the research group were followed and unforced errors were made in the unforced error analysis table according to the region where they were made. All data were collected using simple error analysis table. The obtained data were transferred to the SPSS 22 packet program and the minimum, maximum, mean and standard deviation values for the descriptive statistics were calculated. Mann Withney $U$ test was used to analyze the difference between the categories and the winner and the loser. There was a significant difference between the TPL $(\mathrm{p}<0.05)$, TE, TAE and AEMC values of the winners and loser in the U19 category $(\mathrm{p}<0.05)$. There was a significant difference between the TPL values of the winners and the losers in the U17 category ( $<<0.01$ ). As a result, in the U19 group, the athletes in the mid-court area and in total fewer out-of-mistakes ended up winning the competition.
\end{abstract}

Original Article

Anahtar kelimeler:

Badminton,

basit hata,

milli, müsabaka

\section{competition.}

*Sorumlu Yazar: Beyhan ÖZGÜR, E-mail: beyhanozgur82@hotmail.com, +90 5063145027 


\section{GíRiş}

Badminton, ortasında file bulunan bir kortta, raket ile, kaz tüyünden yapılmış topu rakip alana düşürerek sayı kazanmak amacıyla oynanan olimpik bir spor dalıdır. Sporcular, tek erkekler, tek kadınlar, çift erkekler, çift kadınlar ve karışık çiftler olmak üzere 5 farklı kategoride mücadele ederler. Bu kategoriler 11, 13, 15, 17, 19 yaş altı ve büyükler olmak üzere farklı yaş gruplarında oynanır. Badminton müsabakaları 21 sayılık kazanılmış 2 set üzerinden oynanır (Cümşütoğlu ve Kale, 1994; BWF, 2011).

Badminton, yüksek tempolu, dinamik ve enerjik rallileri ile bilinen dünyanın en hızlı raket sporu olarak kabul edilmektedir (Butterworth, Turner \& Johnstone, 2012). Olimpiyat Oyunlarında yapılan analiz sonuçlarına göre, erkek badminton oyuncularının 1.09 vuruş sayısı/sn ile, 9.1 saniye süren rallilerde 9.9 vuruş gerçekleştirdikleri bildirilmiştir (AbianVicen, Castanedo, Abian \& Sampedro, 2013). Bu vuruşlar, ön kort bölgesinde; net drop, lob ve net kill, orta kort bölgesinde; drive ve push, arka kort bölgesinde; clear, drop, smaç gibi teknikler ile gerçekleştirilir (Larsen, 2006). Ortalama olarak 30-50 dakika süren müsabakalarda, oyuncular 500 den fazla raket savurma ve vuruş gerçekleştirirler (Coşan ve Demir, 2016). Vuruş sayısı özellikle üst düzey turnuvalarda daha fazla artmaktadır. Rio Olimpiyat Oyunlarında, 76.6 servis, 259.9 net drop, 193.1 lob, 105.6 drop, 100.6 smaç, 59.0 clear ve 11.3 drive toplamda 806.4 vuruş tekniği yapıldığını bildirmiştir (Chiminazzo, Barreira, Luz, Saraiva \& Cayres, 2016). Badmintoncular bu zorlu tempolara uyum sağlayabilmek ve galibiyet için, hız, çeviklik, esneklik, dayanıklılık ve güç sınırlarında performans gösterirken, aynı zamanda rakibe karşı tekniksel, taktiksel ve mental durumlarını koruyarak hata sayısını azaltmak zorundadırlar (S, 2016).

Literatürde, genellikle badmintoncuların fiziksel performans seviyelerinin belirlenmesi ve geliştirilmesi ile ilgili çalışmalar görülmekle birlikte (Hotaman, Özgür ve Coşan, 2018), bazı araştırmacılar, müsabaka ortamında yapılan basit hataların analizine yönelik çalışmalara odaklanmıştır. Yadav \& Shukla (2011) tarafından yapılan çalışmada maç kazanan badmintoncuların kaybeden oyunculara göre daha az basit hata yaptıkları bulunmuştur. $\mathrm{Bu}$ hatalar, file hatası (topun fileye takılması) ve aut hatası (topun oyun alanı dişına atılması) olarak değerlendirilmektedir. Pekin Olimpiyat Oyunlarında basit hatalar ile kaybedilen sayıların, erkek oyuncularda \% 41.0, kadın sporcularda ise \% 48.6 olduğu bulunmuştur (Abian-Vicen, Castanedo, Abian \& Sampedro, 2013). Pekin ve Londra Olimpiyat Oyunlarının karşılaştırıldığ çalışmada ise basit hatalar ile kaybedilen sayılar Pekin Oyunlarında \% 41.0 Londra Oyunlarında \% 42.6 olarak bulunmuştur (Abian, Castanedo, Feng \& Abian-Vicen, 2014).

$\mathrm{Bu}$ güne kadar Türk badminton oyuncularının müsabaka ortamında ortamında yaptıkları basit hatalara yönelik herhangi bir çalışma ele alınmamıştır. Bu çalışmada 17 ve 19 yaş grubu erkek milli badmintoncuların maç ortamında yaptıkları basit hataların belirlenmesi, yaş gruplarına ve kazanma durumuna göre karşılaştırılması amaçlanmıştır. 


\section{YÖNTEM}

\section{Çalışma Grubu}

Araştırmaya, U19 kategorisinden yaş ortalamas1 17,44 $\pm 0,51$ y1l olan 18 ve U17 kategorisinden yaş ortalaması 15,55 $\pm 0,51$ y1l olan 20 erkek milli badminton oyuncusu dahil edilmiştir. Çalışma, 13-16 Aralık 2017 tarihlerinde Ankara'da düzenlenen uluslararası gençler badminton turnuvasında (Turkey Junior 2017) gerçekleştirilmiştir.

\section{Basit Hata Analizi}

U17 kategorisinde 31 ve U19 kategorisinde 35 tek erkekler maçı analiz edilmiştir. Basit hata analizi, TH (toplamda yapılan basit hata), KTS (kaybedilen toplam sayı), BKTSY (basit hataların kaybedilen toplam sayıdaki yüzdesi), HÖK (hata ön kort), HOK (hata orta kort), HAK (hata arka kort), HAÖK (hata aut ön kort), HAOK (hata aut orta kort), HAAK (hata aut arka kort), TAH (toplamda aut hatas1), HFÖK (hata file ön kort), HFOK (hata file orta kort), HFAK (hata file arka kort), TFH (Toplamda File Hatası) olarak yapılmıştır (Hotaman, Özgür ve Coşan, 2018).

Tablo 1. Badmintonda basit hata analiz tablosu

\begin{tabular}{|c|c|c|c|c|c|c|c|c|c|c|c|}
\hline \multicolumn{12}{|c|}{ Badmintonda Basit Hata Analiz Tablosu } \\
\hline \multicolumn{6}{|c|}{ Aut Hatas1 } & \multicolumn{6}{|c|}{ File Hatası } \\
\hline \multicolumn{2}{|c|}{ Ön Kort } & \multicolumn{2}{|c|}{ Orta kort } & \multicolumn{2}{|c|}{ Arka kort } & \multicolumn{2}{|c|}{ Ön kort } & \multicolumn{2}{|c|}{ Orta kort } & \multicolumn{2}{|c|}{ Arka kort } \\
\hline $\mathrm{F}$ & $\mathrm{B}$ & $\mathrm{F}$ & $\mathrm{B}$ & $\mathrm{F}$ & $\mathrm{B}$ & $\mathrm{F}$ & $\mathrm{B}$ & $\mathrm{F}$ & $\mathrm{B}$ & $\mathrm{F}$ & $\mathrm{B}$ \\
\hline & & & & & & & & & & & \\
\hline
\end{tabular}

F: Forehand, B: Backhand

Müsabaka sırasında araştırma grubunun maçları izlenmiş ve yapılan basit hatalar, yapıldığı bölgeye göre basit hata analiz tablosuna adet olarak işlenmiştir. Analiz yapan kişi (5 kişi) sayısından fazla maç olduğunda maçlar kamera kaydına alınmış ve daha sonra analiz edilmiştir (Hotaman, Özgür ve Coşan, 2018).

\section{Verilerin Analizi}

Elde edilen veriler SPSS 22 paket programına aktarılmış ve tanımlayıcı istatistikleri için minimum, maksimum, ortalama ve standart sapma değerleri hesaplanmıştır. Verilerin normal dağılama sahip olup olmadılarını anlamak için Shapiro-Wilk testi kullanılmıştır. Normal dağılama sahip olmadığı görülen verilerin analizi Mann Withney U testi ile yapılmıştır. 


\section{BULGULAR}

Tablo 2. 1. sette yapılan basit hataların kategorilere göre karşılaştırılması

\begin{tabular}{lccc}
\hline Parametreler & $\mathbf{U 1 7}$ & $\mathbf{U 1 9}$ & P \\
\cline { 2 - 4 } & $\begin{array}{c}\text { Maç } \\
(\mathbf{n}=\mathbf{3 1})\end{array}$ & $\begin{array}{c}\text { Maç } \\
(\mathbf{n}=\mathbf{3 5})\end{array}$ & 0.061 \\
\hline KTS & $18.3 \pm 4.60$ & $16.8 \pm 4.20$ & 0.802 \\
BKTSY (\%) & $48.2 \pm 19.3$ & $49.5 \pm 21.7$ & 0.557 \\
TH & $8.55 \pm 3.65$ & $8.06 \pm 3.42$ & 0.834 \\
TAH & $4.32 \pm 2.32$ & $4.20 \pm 2.39$ & 0.885 \\
TFH & $4.19 \pm 2.28$ & $4.11 \pm 2.13$ & 0.815 \\
HÖK & $4.03 \pm 2.52$ & $3.82 \pm 2.22$ & 0.628 \\
HOK & $1.35 \pm 1.19$ & $1.74 \pm 1.80$ & 0.795 \\
HAK & $3.06 \pm 2.54$ & $2.71 \pm 1.82$ & 0.748 \\
HAÖK & $2.16 \pm 1.84$ & $1.89 \pm 1.34$ & 0.150 \\
HAOK & $0.42 \pm 0.67$ & $0.77 \pm 1.00$ & 0.662 \\
HAAK & $1.77 \pm 1.54$ & $1.54 \pm 1.29$ & 0.942 \\
HFÖK & $1.87 \pm 1.36$ & $1.94 \pm 1.60$ & 0.962 \\
HFOK & $0.94 \pm 1.03$ & $0.97 \pm 1.09$ & 0.957 \\
HFAK & $1.29 \pm 1.46$ & $1.17 \pm 1.24$ & \\
\hline
\end{tabular}

Tablo 2' de 1. sette yapılan basit hata değerleri incelendiğinde, kategoriler arası anlamlı fark görülmemiştir ( $>00.05)$.

Tablo 3. 2. sette yapılan basit hataların kategorilere göre karşılaştırılması

\begin{tabular}{lccc}
\hline Parametreler & U17 & U19 & P \\
\cline { 2 - 4 } KTS & $\begin{array}{c}\text { Maç } \\
(\mathbf{n}=\mathbf{3 1})\end{array}$ & $\begin{array}{c}\text { Maç } \\
(\mathbf{n}=\mathbf{3 5})\end{array}$ & 0.287 \\
BKTSY (\%) & $18.1 \pm 4.74$ & $16.9 \pm 4.61$ & 0.168 \\
TH & $42.1 \pm 21.1$ & $46.1 \pm 18.0$ & 0.449 \\
TAH & $7.06 \pm 2.64$ & $7.57 \pm 2.75$ & 0.535 \\
TFH & $3.52 \pm 2.23$ & $3.86 \pm 2.01$ & 0.512 \\
HÖK & $3.55 \pm 2.43$ & $3.71 \pm 2.12$ & 0.274 \\
HOK & $3.45 \pm 2.30$ & $3.74 \pm 1.70$ & 0.166 \\
HAK & $1.25 \pm 1.80$ & $1.51 \pm 1.29$ & 0.754 \\
HAÖK & $2.32 \pm 1.66$ & $2.31 \pm 2.01$ & 0.460 \\
HAOK & $1.58 \pm 1.60$ & $2.06 \pm 1.51$ & 0.385 \\
HAAK & $0.61 \pm 1.43$ & $0.49 \pm 0.81$ & 0.406 \\
HFÖK & $1.32 \pm 1.30$ & $1.31 \pm 1.51$ & 0.995 \\
HFOK & $1.87 \pm 1.80$ & $1.69 \pm 1.18$ & 0.409 \\
HFAK & $0.65 \pm 0.79$ & $1.03 \pm 0.95$ & 0.979 \\
\hline
\end{tabular}

Tablo 3' te 2. sette yapılan basit hata değerleri incelendiğinde, kategoriler arası anlamlı fark görülmemiştir ( $\mathrm{p}>0.05)$. 
Tablo 4. Maç genelinde yapılan basit hataların kategorilere göre karşılaştırılması

\begin{tabular}{lccc}
\hline Parametreler & U17 & U19 & P \\
\cline { 2 - 4 } & $\begin{array}{c}\text { Maç } \\
(\mathbf{n = 3 1})\end{array}$ & $\begin{array}{c}\text { Maç } \\
(\mathbf{n = 3 5})\end{array}$ & 0.111 \\
KTS & $36.4 \pm 8.65$ & $33.7 \pm 7.93$ & 0.481 \\
TKTSY (\%) & $44.6 \pm 16.0$ & $47.4 \pm 15.8$ & 0.991 \\
TAH & $15.6 \pm 5.65$ & $15.6 \pm 5.34$ & 0.806 \\
TFH & $7.84 \pm 3.64$ & $8.06 \pm 3.51$ & 0.698 \\
HÖK & $7.74 \pm 4.23$ & $7.83 \pm 3.69$ & 0.591 \\
HOK & $7.48 \pm 4.00$ & $7.57 \pm 3.44$ & 0.260 \\
HAK & $2.61 \pm 2.17$ & $3.25 \pm 2.51$ & 0.698 \\
HAÖK & $5.38 \pm 2.94$ & $5.02 \pm 2.74$ & 0.460 \\
HAOK & $3.74 \pm 2.90$ & $3.94 \pm 2.42$ & 0.385 \\
HAAK & $1.03 \pm 1.87$ & $1.26 \pm 1.50$ & 0.406 \\
HFÖK & $3.10 \pm 1.51$ & $2.86 \pm 1.70$ & 0.995 \\
HFOK & $3.74 \pm 2.55$ & $3.63 \pm 2.40$ & 0.409 \\
HFAK & $1.58 \pm 1.20$ & $2.00 \pm 1.60$ & 0.979 \\
\hline
\end{tabular}

Tablo 4'te maç genelinde yapılan basit hata değerleri incelendiğinde, kategoriler arası anlamlı fark görülmemiştir ( $\mathrm{p}>0.05)$.

Tablo 5. Kazanan ve Kazanamayan badmintoncuların yaptıkları basit hataların karşılaştırılması

\begin{tabular}{|c|c|c|c|c|c|}
\hline Parametreler & & $\begin{array}{c}\text { U19 } \\
\text { Maç (n) } \\
\text { G=22 / M=13 }\end{array}$ & $\mathbf{P}$ & $\begin{array}{c}\text { U17 } \\
\text { Maç (n) } \\
\text { G=10 / M=21 }\end{array}$ & $\mathbf{P}$ \\
\hline \multirow{2}{*}{ KTS } & Kazanan & $29.8 \pm 7.03$ & \multirow{2}{*}{$0.000 * *$} & $28.7 \pm 9.14$ & \multirow{2}{*}{$0.000 * *$} \\
\hline & Kazanamayan & $40.3 \pm 4.09$ & & $40.1 \pm 5.55$ & \\
\hline \multirow{2}{*}{ BKTSY \%) } & Kazanan & $48.2 \pm 16.0$ & \multirow{2}{*}{0.682} & $52.6 \pm 17.2$ & \multirow{2}{*}{0.069} \\
\hline & Kazanamayan & $45.9 \pm 16.0$ & & $40.8 \pm 14.2$ & \\
\hline \multirow{2}{*}{ TH } & Kazanan & $14.1 \pm 4.87$ & \multirow{2}{*}{$0.025^{*}$} & $14.6 \pm 5.92$ & \multirow{2}{*}{0.524} \\
\hline & Kazanamayan & $18.1 \pm 5.32$ & & $16.1 \pm 5.60$ & \\
\hline \multirow{2}{*}{ TAH } & Kazanan & $7.05 \pm 2.60$ & \multirow{2}{*}{$0.035^{*}$} & $7.30 \pm 3.12$ & \multirow{2}{*}{0.481} \\
\hline & Kazanamayan & $9.77 \pm 4.24$ & & $8.10 \pm 3.91$ & \\
\hline \multirow{2}{*}{ TFH } & Kazanan & $7.50 \pm 3.71$ & \multirow{2}{*}{0.503} & $7.20 \pm 3.70$ & \multirow{2}{*}{0.750} \\
\hline & Kazanamayan & $8.38 \pm 3.75$ & & $8.00 \pm 4.52$ & \\
\hline \multirow{2}{*}{ HÖK } & Kazanan & $6.86 \pm 3.12$ & \multirow{2}{*}{0.101} & $6.50 \pm 3.59$ & \multirow{2}{*}{0.371} \\
\hline & Kazanamayan & $8.76 \pm 3.76$ & & $7.95 \pm 4.18$ & \\
\hline \multirow{2}{*}{ HOK } & Kazanan & $3.00 \pm 2.81$ & \multirow{2}{*}{0.184} & $2.50 \pm 1.64$ & \multirow{2}{*}{0.863} \\
\hline & Kazanamayan & $3.69 \pm 1.93$ & & $2.66 \pm 2.41$ & \\
\hline \multirow{2}{*}{ НАК } & Kazanan & $4.68 \pm 2.88$ & \multirow{2}{*}{0.216} & $5.30 \pm 2.71$ & \multirow{2}{*}{0.831} \\
\hline & Kazanamayan & $5.61 \pm 2.50$ & & $5.42 \pm 3.10$ & \\
\hline \multirow{2}{*}{ НАÖК } & Kazanan & $3.59 \pm 2.01$ & \multirow{2}{*}{0.329} & $3.40 \pm 1.95$ & \multirow{2}{*}{0.983} \\
\hline & Kazanamayan & $4.54 \pm 2.98$ & & $3.90 \pm 3.30$ & \\
\hline \multirow{2}{*}{ HAOK } & Kazanan & $0.91 \pm 1.34$ & \multirow{2}{*}{$0.039 *$} & $1.00 \pm 1.15$ & \multirow{2}{*}{0.614} \\
\hline & Kazanamayan & $1.85 \pm 4.62$ & & $1.05 \pm 2.15$ & \\
\hline \multirow{2}{*}{ НАAK } & Kazanan & $2.55 \pm 1,47$ & \multirow[b]{2}{*}{0.222} & $3.00 \pm 1.49$ & \\
\hline & Kazanamayan & $3.38 \pm 1.98$ & & $3.14 \pm 1.55$ & 0.897 \\
\hline & Kazanan & $3.27 \pm 1.88$ & & $3.10 \pm 1.96$ & \\
\hline HFÖK & Kazanamayan & $4.23 \pm 3.08$ & 0.400 & $4.04 \pm 2.78$ & 0.290 \\
\hline & Kazanan & $2.09 \pm 1.82$ & & $1.50 \pm 1.43$ & \\
\hline HFOK & Kazanamayan & $1.85 \pm 1.21$ & 0.944 & $1.62 \pm 1.11$ & 0.791 \\
\hline & Kazanan & $2.14 \pm 2.23$ & & $2.30 \pm 1.82$ & \\
\hline HFAK & Kazanamayan & $2.23 \pm 1.48$ & 0.520 & $2.29 \pm 2.41$ & 0.682 \\
\hline
\end{tabular}

$\mathbf{p}<\mathbf{0 . 0 5 *}, \mathbf{p}<\mathbf{0 . 0 1 * *}$, G: kazanan, M: Kazanamayan 
Tablo 5' te U17 ve U19 kategorilerinde kazanan ve kazanamanyan sporcuların KTS değerleri arasında anlamlı fark bulunurken ( $\mathrm{p}<0.01)$, U19 kategorisinde kazanan ve kazanamanyan sporcuların TH, TAH ve HAOK değerleri arasında anlamlı fark bulunmuştur $(\mathrm{p}<0.05)$.

\section{TARTIŞMA}

Bu çalışmada U17 ve U19 milli erkek badmintoncuların müsabaka sırasında yaptıkları basit hatalar karşılaştırılmıştır. Literatürdeki basit hataları incelemeye yönelik çalışmalar oldukça sınırlıdır. Bu araştırmalar çoğunlukla maç genelinde yapılan basit hataları incelemeye yönelik gerçekleştirilmiştir. Çalışmamızda maç genelinde yapılan basit hatalara ilaveten, set durumuna göre (1. set, 2. set ve 3. set) oyun alanı bölgelerine yönelik (ön kort, orta kort, arka kort) ve aut / file hatası durumuna göre basit hata analizi de yapılmıştır. Çalışma sonuçlarına göre U17 ve U19 yaş gruplarının 1. sette, 2. sette ve maç genelinde yaptıkları tüm basit hata değerleri benzer bulunmuştur ( $p>0.05$ ). Laffaye, Phomsoupha \& Dor (2015) tarafindan yapılan çalışmada, Barselona, Atlanta, Sidney, Atina, Pekin ve Londra Olimpiyat Oyunlarının final müsabakalarında yapılan basit hata değerleri \% 42.00 olarak bulunmuştur. Abian, Castanedo, Feng \& Abian-Vicen (2014) basit hatalar ile kaybedilen say1lar1, Pekin

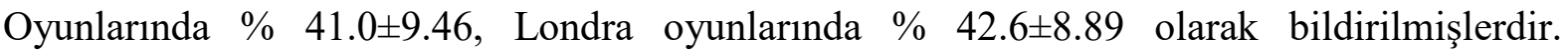

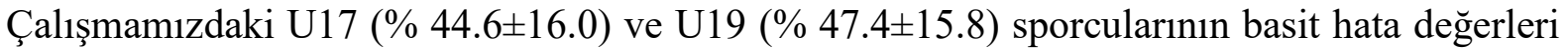
literetürden yüksek görülmektedir. Bu farkın, Olimpiyat Oyunlarına katılma hakkı kazanan sporcuların tekniksel, taktiksel ve psikolojik olarak daha iyi seviyede olmalarından kaynaklandığı düşünülmektedir. Basit hataların, genel olarak dikkat, konsantrasyon, teknik veya taktiksel farkındalık eksikliğinden kaynaklandığı belirtilmektedir (Yadav \& Shukla, 2011). Bununla birlikte basit hataların fiziksel performans seviyesi ile ilişkili olduğunu gösteren çalışmalar da mevcutur (Omveer, 2017; Hotaman, Özgür ve Coşan, 2018).

Kazanan ve kaybeden oyuncuların basit hata değerleri incelendiğinde, U17 ve U19 grubunda kazanan oyuncuların kazanamanyan oyunculara göre KTS (kaybedilen toplam sayı), değerleri anlamlı olarak daha az bulunmuştur $(\mathrm{p}<0.01)$. Bu sonuçlar maçların uzatmalara gitmeden sonuçlandığını göstermektedir. U19 grubunda kazanan oyuncuların kazanamanyan oyunculara göre TH (toplam hata), TAH (toplam aut hatası) ve HAOK (hata aut orta kort) değerleri anlamlı olarak daha az bulunmuştur $(\mathrm{p}<0.05)$. Tong \& Hong (2000) tarafindan

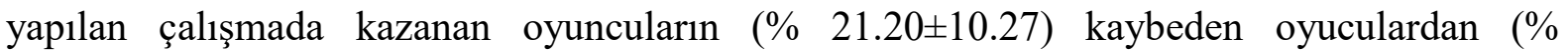
$25.30 \pm 8.23$ ) daha az basit hata yaptıkları görülmüştür. Diğer bir çalışmada daha az basit hata yapan sporcuların \% 73' ü müsabakaları galip olarak bitirmiştir (Cabello-Manrique \& González-Badillo, 2003). Dobson (2001) elit-altı oyuncuların elit oyunculara göre daha yüksek bir basit hata yüzdesi gösterdiklerini bildirirken, Har (2007) kazanan ve kaybeden oyuncuların basit hataları arasında anlamlı fark olmadığını bildirmiştir (Ming, Keong \& Ghosh, 2008). Bu çalışma sonuçlarına göre, U19 grubunda özellikle daha az aut hatası yapan oyuncuların maç kazanabilmeleri için önemli bir avantaj elde ettikleri görülmektedir. U17 grubunda kazanan ve kazanamanyan oyuncuların basit hata değerlerinde anlamlı fark bulunmamıştır $(\mathrm{p}>0.05)$. Bu bulgular, basit hataların özellikle üst yaş grupları ve elit seviyede oynayan badmintontoncuların müsabakalarında, maç sonuçlarını daha belirleyici olduğunu göstermektedir. 


\section{SONUÇ}

U17 ve U19 gruplarının müsabaka ortamında yaptıkları tüm basit hata değerlerinin benzer olduğu görülürken, U19 grubunda, orta kort bölgesinde ve toplamda daha az aut hatas1 yapan sporcuların müsabakaları galip olarak bitirdikleri söylenebilir. Çok toplu besleme çalışmaları ile orta kort bölgesindeki antrenmanların kapsamı arttırılarak, basit hataların azaltılmasına katkı sağlanabilir. Gelecek çalışmalarda teknik vuruşlara göre basit hata analizi yapılması önerilmektedir.

\section{KAYNAKLAR}

Abian, P., Castanedo, A., Feng, XQ., \& Abian-Vicen, J. (2014). Notational comparison of men's singles badminton matches between Olympic Games in Beijing and London. International Journal of Performance Analysis in Sport, 14(1), 42-53.

Abian-Vicen, J., Castanedo, A., Abian, P., \& Sampedro, J. (2013). Temporal and notational comparison of badminton matches between men's singles and women's singles. International Journal of Performance Analysis in Sport, 13(2), 310-320.

Butterworth, DA., Turner, J.D., \& Johnstone, A.J. (2017). Coaches' perceptions of the potential use of performance analysis in badminton. International Journal of Performance Analysis in Sport, 12(2), $452-467$.

BWF. (2011). BWF coaches' manual level 1, Badminton World Federation, Kuala Lumpur, Malaysia.

Cabello-Manrique, D., \& González-Badillo, J.J. (2003). Analysis of the characteristics of competitive badminton. British journal of sports medicine, 37(1), 62-66.

Chiminazzo, J.G.C., Barreira, J., Luz, L.S.M., Saraiva, W.C., \& Cayres J.T. (2018). Technical and timing characteristics of badminton men's single: comparison between groups and play-offs stages in 2016 Rio Olympic Games. International Journal of Performance Analysis in Sport, 18(2), 245-254.

Cosan, F., ve Demir, G. (2016). Sporda atletik hazırlı̆̆ı denetimi ve değerlendirilmesinde yenilikçi yaklaşımlar. Razgrat-Bulgaria: Razgrat-polygraph Ltd.

Cümşütoğlu, R.M. ve Kale, R. (1994). Uçan tüytop badminton. İstanbul: Başak Ofset. .

Hotaman, F., Özgür, B. ve Coşan, F. (2018). 17 yaş grubu milli badmintoncuların müsabaka sırasında yaptıkları basit hatalar ile fiziksel performansları arasındaki ilişki, Spor Bilimleri Araştırmaları Dergisi, 3(1), (1), 73-84.

Laffaye, G., Phomsoupha, M., \& Dor, F. (2015). Changes in the game characteristics of a badminton match: a longitudinal study through the olympic game finals analysis in men's singles. J Sports Sci Med, 14(3), 584-590.

Larsen, K. (2006). Badminton europe coach education level one. Denmark: Broendby.

Ming, C.L., Keong, C.C., \& Ghosh, A.K. (2008). Time motion and notational analysis of 21 point and 15 point badminton match play. International journal of sports science and engineering, 2(4), 216-222.

Omveer. (2017). A study on prediction of playing ability in badminton from selected anthropometrical physical and physiological characteristics among inter collegiate players. International Journal of Advanced Research and Development, 2(5), 50-54. 
S, M. (2016). Relative importance of anthropometric biomotor and skill performance to playing ability of college badminton players. International journal of physical education, sports and health, 3(2), 156158.

Tong, Y.M., \& Hong, Y. (2000). The playing pattern of world's top single badminton players. In Youlian H, DP Johns, R Sanders (Eds). 18th International Symposium on Biomechanics in Sports (p, 1-6). Hong Kong.

Yadav, S.K., \& Shukla, Y.M. (2011). Analysis of unforced errors in relation to performance in singles in badminton. International Journal of Physical Education, 4(2), 117-119. 